\title{
Potassium-titanyl-phosphate laser assisted robotic partial nephrectomy in a porcine model: can robotic assistance optimize the power needed for effective cutting and hemostasis?
}

\author{
Ronald S. Boris · Daniel Eun • Akshay Bhandari • \\ Kathryn Lyall $\cdot$ Mahendra Bhandari $\cdot$ Craig Rogers $\cdot$ \\ Osama Alassi · Mani Menon
}

Received: 10 June 2007 / Accepted: 15 June 2007 / Published online: 3 August 2007

(C) Springer London 2007

\begin{abstract}
A potassium-titanyl-phosphate (KTP) laser through robotic endo-wrist instrument has been evaluated as an ablative and hemostatic tool in robotic assisted laparoscopic partial nephrectomy (RALPN). Ten RALPN were performed in five domestic female pigs. The partial nephrectomies were performed with bulldog clamping of the pedicle. Flexible glass fiber carrying 532-nm green light laser was used through a robotic endowrist instrument in two cases. Power usage from 4 to $10 \mathrm{~W}$ was tested. The laser probe was explored both as a cutting knife and for hemostasis. The pelvicalyceal system was closed with a running suture. Partial nephrectomies using KTP laser were performed without complications. Mean operative times and warm ischemia times for laser cases were 96 and $18 \mathrm{~min}$, respectively. Mean estimated blood loss was $60 \mathrm{ml}$ compared with $50 \mathrm{ml}$ for non-laser cases. Complete hemostasis with the laser alone could be achieved with a power of $4 \mathrm{~W}$ and was found to be effective. In our hands the laser fiber powered up to $10 \mathrm{~W}$ was not effective as a quick cutting agent. Histopathologic analysis of the renal remnant revealed a cauterized surface effect with average laser penetration depth less than $1 \mathrm{~mm}$ and minimal surrounding
\end{abstract}

R. S. Boris $(\bowtie) \cdot$ D. Eun · A. Bhandari · M. Bhandari ·

C. Rogers - M. Menon

Vattikuti Urology Institute,

Henry Ford Health System, Detroit, MI 48202, USA

e-mail: rboris1@hfhs.org

K. Lyall

Intuitive Surgical, Sunnyvale, CA USA

O. Alassi

Department of Pathology,

Henry Ford Hospital System, Detroit, MI USA cellular injury. The new robotic endowrist instrument carrying flexible glass fiber transmitting 532-nm green light laser is a useful addition to the armamentarium of the robotic urologic setup. Its control by the console surgeon enables quicker and more complete hemostasis of the cut surface in renal sparing surgery using a porcine model. Histologically proven lased depth of less than $1 \mathrm{~mm}$ suggests minimal parenchyma damage in an acute setting. Laser application as a cutting agent, however, requires further investigation with interval power settings beyond the limits of this preliminary study. We estimate that effective cutting should be possible with a setting lower than traditionally recommended for solid organs.

Keywords Laparoscopy · Partial nephrectomy · KTP laser $\cdot$ Robot $\cdot$ Porcine model

\section{Introduction}

Despite recent trends favoring minimal techniques for nephron sparing surgery, open partial nephrectomy remains the reference standard against which all minimally invasive techniques should be evaluated [1]. Laparoscopic partial nephrectomy is a complex surgical undertaking and demands advanced laparoscopic skills in accomplishing time-sensitive tasks of tumor excision, hemostasis, and reconstruction of the resulting collecting system defect. This involves extensive intracorporeal suturing within permissible ischemia times, because this predicts subsequent recovery of kidney function [2].

Despite the recent development of various techniques to reduce intraoperative bleeding in a time-effective manner, achieving renal parenchymal hemostasis during and after the procedure remains a significant challenge. Laser 
induced thermotherapy is the latest thermoablation technology that is gaining widespread acceptance. Encouraging outcomes have been reported in the application of the potassium-titanyl-phosphate (KTP) laser powered at $80 \mathrm{~W}$ for laparoscopic partial nephrectomy in the survival calf model [3].

The da Vinci surgical system has been successfully integrated into radical prostate surgery and has recently emerged as having potential in upper tract surgery. It has advantages over standard laparoscopy including a precise stereoscopic vision, six-degrees of wristed motion, tremor filtering, and motion scaling. We recently investigated the application of a surgical robot for partial nephrectomy in a non-survival porcine model.

We investigated its function as a laser knife for parenchymal shearing and as a hemostatic tool when spray painted on the cut surface of the renal remnant following removal of the diseased kidney. In addition the utility and feasibility of using new micro wrist instrument carrying the 532-nm green light laser fiber controlled by the console surgeon was also evaluated.

\section{Materials and methods}

We have previously reported our technique and initial experience of RALPN series [4]. Given the purported advantages of the da Vinci S system, we hypothesized that this approach may expedite excision, hemostasis, and reconstruction during nephron sparing surgery thereby decreasing operative time, blood loss, and ischemic time.

In an Animal Care and Use Committee-approved study, robotic assisted laparoscopic partial nephrectomy (RALPN) was performed using a transperitoneal approach in five non-survival female pigs (weight $70-100 \mathrm{~kg}$ ). A total of ten partial nephrectomies were performed with an additional four completion nephrectomies for a total of 14 procedures. Pigs were euthanized immediately after completion of the second nephrectomy using a standard proto- col. In this series two of the ten partial nephrectomies were performed with a KTP laser. All intraoperative data were prospectively collected and recorded in an electronic database. Anesthesia records were obtained from the veterinary anesthesiologist.

The laser instrument was an EndoWrist reposable instrument designed for use with the disposable Laserscope green light laser fiber. The laser tool acts as a conduit through which a flexible laser fiber is inserted and directed using the da Vinci system. Potential uses include cutting, cauterizing, and coagulation during laparoscopic surgical procedures. Salient features of this instrument include, tool shaft, wrist, and outer diameter equivalent to existing $5 \mathrm{~mm}$ robotic instruments, a tip diameter of $2.5 \mathrm{~mm}$, and a $1.5 \mathrm{~mm}$ lumen running the entire length of the tool. A disposable $4 \mathrm{Fr}$ catheter $(70 \mathrm{~cm}$ length) is fed through the lumen. The laser fiber is then fed through the catheter ready for use. Disposable accessories limit usage to up to $10 \mathrm{~W}$ for the instrument (Fig. 1).

\section{Positioning and port placement}

After induction of general anesthesia, each pig was placed in a modified flank position. Pneumoperitoneum was established with a Veress needles. A 12-mm port was placed on the anterior axillary line at the level of the hilum. A second port was placed under vision four finger breadths lateral to the umbilicus at the level of the hilum. The $8-\mathrm{mm}$ robotic ports were placed in diamond configuration 4-5 finger breadths between the two 12-mm ports. Two addition ports were placed: a 10-mm robotic port (specifically for the da Vinci $S$ fourth arm) placed two fingers medial to the costal margin and a 12-mm assistant port mid clavicular line at the superior level of the costal margin (Fig. 2).

\section{Technique of partial nephrectomy with KTP laser}

Initial dissection was carried out using the Endowrist Prograsp forceps and a Monopolar hook cautery. After dissect-
Fig. 1 Overview of laser equipment and generator. a Endowrist laser instrument with laser fiber protruding (inset). b. Aura XP generator
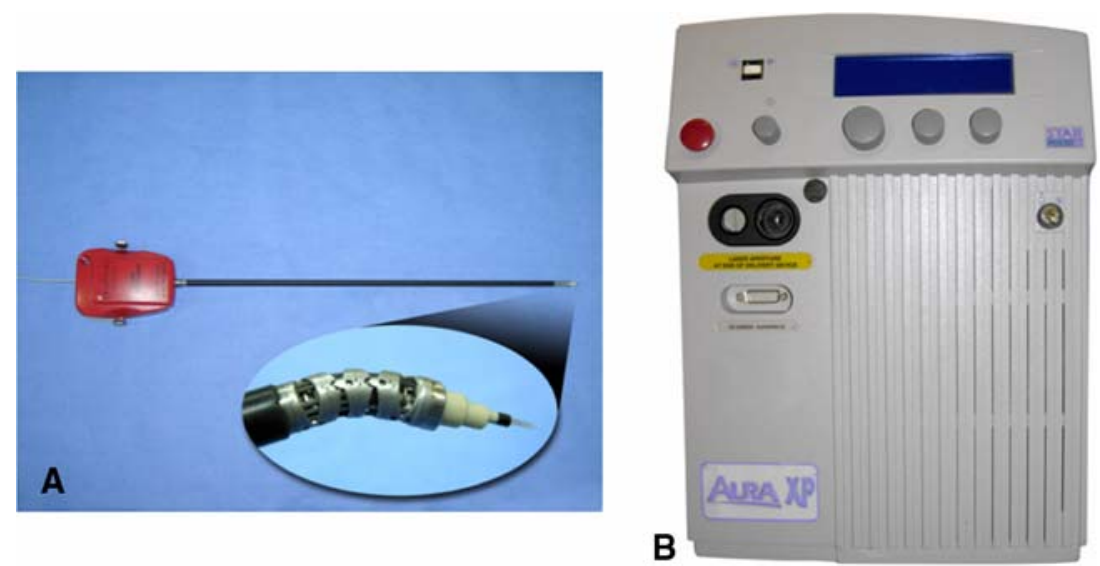


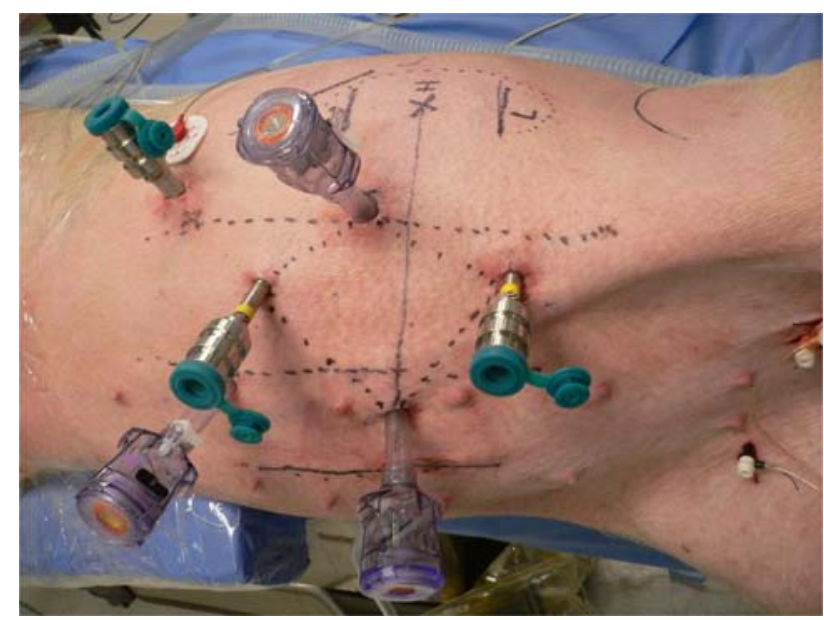

Fig. 2 Diamond configuration of port placement for robotic renal surgery. Note orientation of pig is such that head is to left and foot is to right on photo

ing the renal hilum a laparoscopic bulldog was introduced by the bedside assistant and deployed on the hilum using the Endowrist Prograsp forceps. The laser was then assembled and the laser instrument was deployed through the right robotic arm. A 200-micron fiber with a $532 \mathrm{~nm}$ wavelength with generator power setting of $8 \mathrm{~W}$ was activated. The fiber was held $2 \mathrm{~mm}$ beyond the distal end of the articulating instrument by the bedside assistant as the console surgeon directed the lasing probe.

In the first case the laser was used as a cutting agent by holding the fiber roughly $1-2 \mathrm{~mm}$ from the renal parenchyma. Laser cutting was cumbersome and timeconsuming despite maximizing generator power to $10 \mathrm{~W}$. Therefore the Endowrist Monopolar hot shears were used to complete the polar nephrectomy. The monopolar feature was used to score the renal capsule and the cold scissors were used to complete resection. Next, the laser instrument was re-introduced in the right hand and held 3-4 mm from the renal bed for approximately $3 \mathrm{~min}$, painting from lateral to medial and superior to inferior, until the entire surface was coagulated (Fig. 3). The field was irrigated and there was no visible bleeding on the renal surface. Next using robotic needle drivers running 2-0 Vicryl sutures were placed to close the collecting system. A sutured bolster was then applied. A gross photo of the coagulated surface of the renal remnant ex vivo can be seen in Fig. 4.

The second partial nephrectomy was performed in a similar fashion. Again, the laser failed to impress as a cutting agent and was quickly abandoned and the instrument was exchanged for the Endowrist Monopolar hot shears. The technique followed for partial nephrectomy was the same as described previously. The laser spray was again applied to the cut surface of the renal remnant. The clamp was then removed and one small sinus pumper was observed.

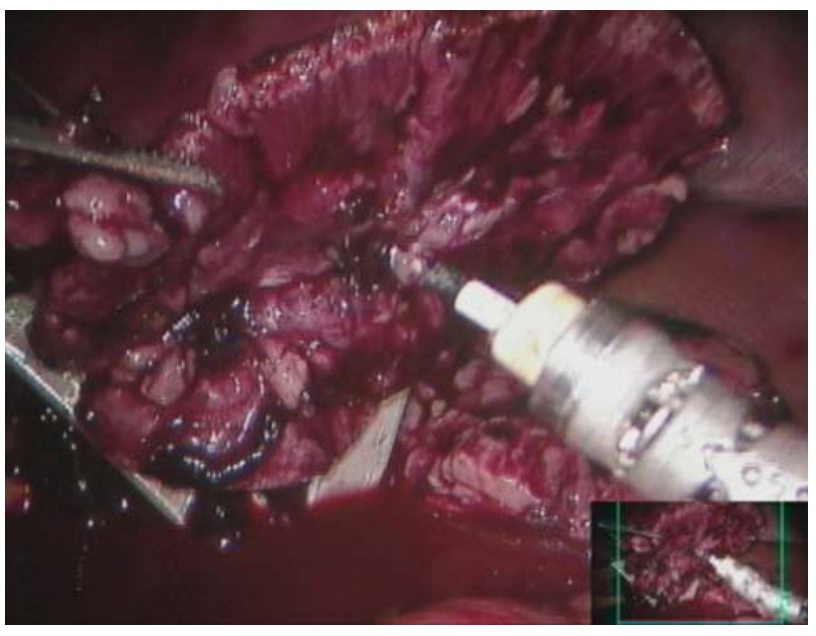

Fig. 3 Robotic KTP laser painting cut surface of renal remnant

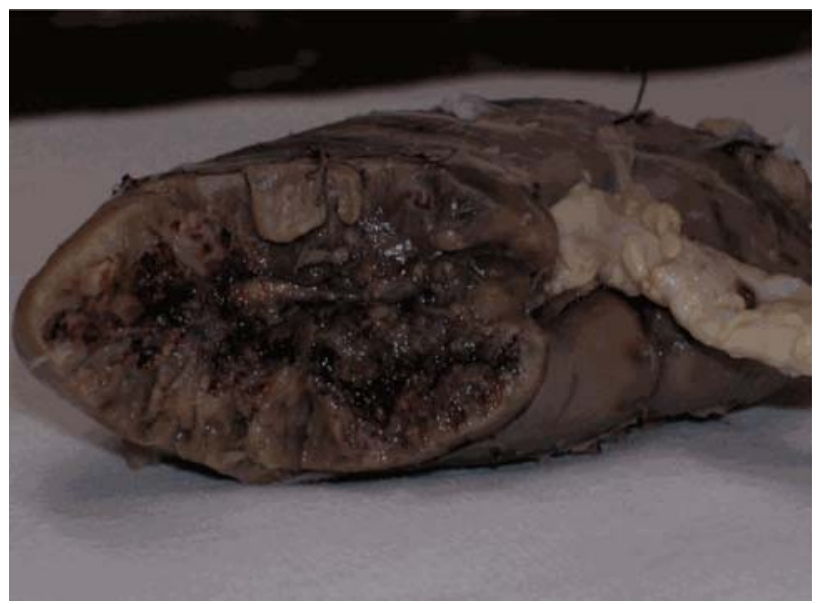

Fig. 4 Gross pathology of renal remnant showing lased cut surface

Extended spot laser application for under $90 \mathrm{~s}$ effectively controlled bleeding without replacing pedicle clamp. A single running 2-0 Vicryl suture was used to close the pelvicalyceal system. No bolster was applied in the second case.

\section{Results}

We performed ten partial nephrectomies in five non-survival pigs. The average time for port placement was $26 \mathrm{~min}$ and the average docking time was 6 min. The KTP laser was used in two cases. General laser information is displayed in Table 1. A comparison of mean operative times, mean warm ischemia times, and mean blood loss is detailed in Table 2. Warm ischemia approached but did not achieve statistical difference in the laser group. Mean blood loss and operative times were comparable between the two groups. $P$ values for all compared categories were determined using the student $t$-test assuming unequal variances. 
Table 1 General laser information KTP laser

\begin{tabular}{llll}
\hline & RLPN & $\begin{array}{l}\text { RLPN with } \\
\text { KTP laser }\end{array}$ & $P$ value \\
\hline $\begin{array}{l}\text { Number of partial } \\
\text { nephrectomies }\end{array}$ & 8 & 2 & - \\
$\begin{array}{c}\text { Mean warm ischemia time } \\
\text { (min), (range) }\end{array}$ & $35(28-48)$ & $18(13-23)$ & 0.054 \\
$\begin{array}{c}\text { Mean operative time } \\
(\text { min), (range) }\end{array}$ & $97(36-175)$ & $96(71-120)$ & 0.969 \\
$\begin{array}{c}\text { Mean estimated blood } \\
\text { loss ml, (range) }\end{array}$ & $50(30-70)$ & $60(50-70)$ & 0.912 \\
\hline
\end{tabular}

Notably, in all partial nephrectomies, the warm ischemia time was $48 \mathrm{~min}$ or less. In the first laser case a running suture for collecting system closure and a sutured bolster for the renal parenchyma was applied after the hilar clamp was released. In the second case only the collecting system was sutured and spot laser coagulation was briefly utilized to control small arterial surface bleeding. The hemostasis was complete and did not require reclamping of the vessels or any additional hemostatic measure. Prior to euthanasia of the pigs the renal fossa was again inspected and showed no evidence of hematoma or bleeding from the remaining surface of the kidney.

The gross appearance of the resection surface shown in Fig. 4 reveals areas of laser coagulation. Histologic sections from the laser-coagulated surface show an average of $1 \mathrm{~mm}$ thickness of loss of substance with loss of cellular detail and nuclear pyknosis in the tubules (Fig. 5). In focal areas the thickness of thermal injury extended up to $3 \mathrm{~mm}$. These areas show more pronounced interstitial hemorrhage and probably represent application of the laser for longer times over bleeding spots. Below this damaged zone, evidence exists of interstitial hemorrhage extending $5 \mathrm{~mm}$ below the cut surface of the kidney.

\section{Discussion}

An increasing number of early renal cell carcinomas qualifying for nephron-sparing surgery has triggered enthusiasm and refinement of the techniques of partial nephrectomy through open and laparoscopic routes. The conventional open and laparoscopic partial nephrectomy series have

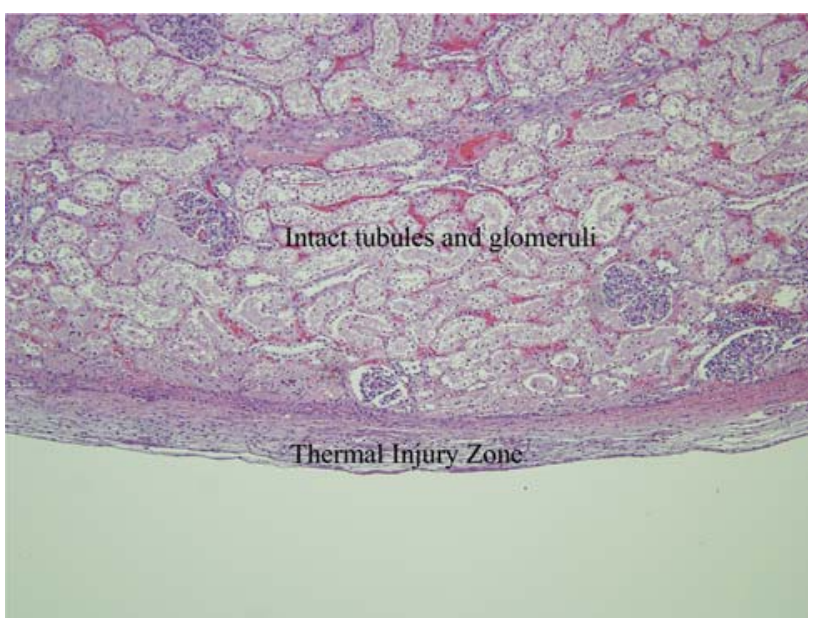

Fig. 5 Perpendicular section of the painted surface of the kidney taken at $10 \times$ magnification showing less than $1 \mathrm{~mm}$ zone of thermal injury. Underlying zone of viable tubules and glomeruli is viable with some vacuolar changes of the tubular epithelium. Entire frame pictured is $2 \mathrm{~mm}$ in height

shown excellent renal functional and oncological outcomes at both 5 and 10-year follow up, respectively [5]. The laparoscopic surgeons have been aiming to keep warm ischemia times below $30 \mathrm{~min}$ to contain the physiologic consequences of extended warm ischemia [6]. The use of tissue sealants [7, 8], radiofrequency [9], lasers [10,11], water jet [12], and microwave [13] in addition to suturing is being investigated for achieving hemostasis during laparoscopic partial nephrectomy.

While recognizing earlier attempts of robot-assisted partial nephrectomy $[4,14]$ we hypothesize that the versatility of the robot could help the surgeon comfortably perform partial nephrectomy within the permissible warm ischemia time. Previous investigations have demonstrated the feasibility of a variety of laser energies for simultaneous cutting and coagulating the renal parenchyma in animal studies and in human subjects during LPN $[10,11,15]$. In this preliminary study we tested the utility of KTP laser as a cutting and hemostatic tool in porcine model.

We used KTP laser, which has a wavelength of $532 \mathrm{~nm}$ resulting in 200 times higher light absorption in hemoglobin than the Nd:YAG laser and a tissue penetration of only $0.8 \mathrm{~mm}$. The selective uptake of KTP laser energy by hemoglobin makes it an appropriate tool for hemostasis [3]. In this study, using a porcine model we investigated the use of a prototype laser instrument for use with the DaVinci $S$ surgical system in robotic partial nephrectomy. This instrument was designed to transmit low power (up to $15 \mathrm{~W}$ ), and with it we explored its likely application both as a laser knife and a laser spray for the purpose of hemostasis. Our familiarity with the KTP laser in surgery for benign prostatic hypertrophy may have facilitated the immediate level of comfort. One explanation for our greater success in laser 
coagulation versus laser cutting is that coagulation and the resulting hemostasis occurs at a lower temperature than vaporization, therefore use of a lower power laser may be appropriate [16]. The reason for accepting the peak power of the laser below the recommendation for solid organ transection is based on expert theories stating that the unavoidable tremor of hand held lasers causes the beam to scatter, therefore any particular volume of tissue is exposed to the laser for a reduced period of time requiring the power to be increased accordingly to achieve the same temperature rise [17]. The potential steadiness of the laser applied with the da Vinci S may facilitate both cutting and coagulation at a much lower power. Use of the laser in this setting is unique because it can be used by the console surgeon.

Contrary to our expectation we observed the process of resection of the renal mass was tedious up to $10 \mathrm{~W}$. In contrast with previous reports, smoke was not a visual deterrent in our experience, probably because the power used was much less than used earlier [15]. Potentially, with all hand tremor eliminated in robotics, the laser may become a more precise and effective tool at wattage between 10 $40 \mathrm{~W}$. We have demonstrated that KTP can achieve effective hemostasis in a very short duration at low power but requires power higher than $10 \mathrm{~W}$ to achieve effective cutting. Further studies are needed to establish it as an very effective cutting tool with the optimum wattage.

Limitations of our study included our small porcine cohort dedicated to KTP use and the lack of ex vivo retrograde pyelography. The delayed consequences of the interstitial hemorrhage seen on histologic analysis are not known at this point. Chronic survival study is suggested to analyze the long-term effects of KTP laser on renal parenchyma.

\section{Conclusions}

The KTP green light laser is a welcome addition to the armamentarium of the robotic surgeon. We found it to be an very effective tool for hemostasis of the renal surface, including large cut vessels, facilitating lower warm ischemia time in robotic partial nephrectomy. Histologically proven lased depth less than $1 \mathrm{~mm}$ suggests minimal injury beyond laser target. In its present state of development it is an ineffective knife, which is likely to adversely effect warm ischemia time. A larger cohort will be required to confirm these preliminary results.

\section{References}

1. Aron M, Gill IS (2007) Minimally invasive nephron-sparing surgery (MINSS) for renal tumours Part II: probe ablative therapy. Eur Urol 51:348-357

2. Caruso RP, Phillips CK, Kau E, Taneja SS, Stifelman MD (2006) Robot assisted laparoscopic partial nephrectomy: initial experience. J Urol 176:36-39

3. Moinzadeh A, Gill IS (2005) Potassium-titanyl-phosphate laser laparoscopic partial nephrectomy without hilar clamping in the survival calf model. J Urol 174:1110-1114

4. Kaul S, Laungani R, Sarle R et al (2006) Da Vinci-assisted robotic partial nephrectomy: technique and results at a mean of 15 months of follow-up. Eur Urol 51:186-192

5. Lane BR, Gill IS (2007) 5-Year outcomes of laparoscopic partial nephrectomy. J Urol 177:70-74

6. Thompson RH, Frank I, Blute ML et al (2007) The impact of ischemia time during open nephron sparing surgery on solitary kidneys: a multi- institutional study. J Urol 177:471-476

7. Morey AF (2005) Improved hemostasis during laparoscopic partial nephrectomy using gelatin matrix thrombin sealant. J Urol 174:1857

8. Gill IS, Ramani AP, Spaliviero M et al (2005) Improved hemostasis during laparoscopic partial nephrectomy using gelatin matrix thrombin sealant. Urology 65:463-466

9. Hegarty NJ, Gill IS (2006) Probe-ablative nephron-sparing surgery: cryoablation versus radiofrequency ablation. Urology 68:713

10. Anderson JK, Baker MR, Lindberg G, Cadeddu JA (2006) Largevolume laparoscopic partial nephrectomy using the potassium-titanyl-phosphate (KTP) laser in a survival porcine model. Eur Urol 51:749-754

11. Lotan Y, Gettman MT, Ogan K, Baker LA, Cadeddu JA (2002) Clinical use of the Holmium:YAG laser in laparoscopic partial nephrectomy. J Endourol 16:289-292

12. Shekarriz H, Shekarriz B, Upadhyay J, Burk C, Wood DP Jr, Bruch HP (2000) Hydro-jet assisted laparoscopic partial nephrectomy: initial experience in a porcine model. J Urol 163:1005-1008

13. Terai A, Ito N, Yoshimura K et al (2004) Laparoscopic partial nephrectomy using microwave tissue coagulator for small renal tumors: usefulness and complications. Eur Urol 45:744-748

14. Caruso RP, Phillips CK, Kau E, Taneja SS, Stifelman MD (2006) Robot assisted laparoscopic partial nephrectomy: initial experience. J Urol 176:36-39

15. Hindley RG, Barber NJ, Walsh K, Petersen A, Poulsen J, Muir GH (2006) Laparoscopic partial nephrectomy using the potassium titanyl phosphate laser in a porcine model. Urology 67:1079-1083

16. Niemz M (2002) In laser-tissue interactions. Fundamentals and applications, 3rd edn. Springer, NY, 78

17. Katzir A (1993) In lasers and optical fibers in medicine. Academic, NY, 74 